\title{
Effective Integration of Gamification and Learning Management Systems for Creating Gamified Learning Arrangements
}

\author{
Bartel, Alexander ${ }^{\mathrm{a}}$; Hagel, Georg ${ }^{\mathrm{a}}$ and Wolff, Christian ${ }^{\mathrm{b}}$
}

${ }^{\mathrm{a}}$ Faculty of Computer Science, Kempten University of Applied Sciences, Germany,

${ }^{\mathrm{b}}$ Faculty of Languages, Literature, and Cultures, University of Regensburg, Germany.

\begin{abstract}
This paper describes ongoing research concerning the effective development of gamified learning arrangements. It shows the necessity for more standardization of the design of such arrangements and reveals potential ways in order to support this need with the use of a domain-specific modeling language. The latter is used by lecturers for designing and exchanging models of gamified learning arrangements and provides the possibility to automatically generate a working learning management system using a language specific generator. The generated learning management system can directly be used by learners and furthermore allows to track the individual learning process for both learners and lecturers.
\end{abstract}

Keywords: Gamification; Domain-Specific Modeling; Learning Management System; Learning. 


\section{Introduction}

The use of game design elements in learning contexts has gained high popularity recently. According to Deterding et al. (2011), the hype about gamification started in the second half of 2010 when the term was coined and has "institutionalized itself as the common household term" (p. 9). Deterding et al. (2011) provide a widely accepted definition for the concept and describe gamification as "the use of game design elements in non-game contexts" (p. 10). Since the creators of this definition characterize it to be on a generic level, the following definition for gamification in learning contexts is suggested for this contribution: "Gamification in learning contexts is described as a concept which integrates game design elements and processes into learning activities in order to increase learning motivation and thereby changes the behavior of learners" (Bartel \& Hagel, 2016, p. 6).

Several researchers (Deterding et al., 2013; Dicheva \& Dichev, 2016; Nah et al., 2014; Rughinis, 2013) argue that there is little research on how to

- systematically enrich learning activities with elements of gamification,

- $\quad$ bring them effectively into practice and

- be able to evaluate their impact on learning.

Meanwhile, some systematic approaches exist (e.g. Danelli, 2015; Deterding, 2015; Kapp, 2014; Marache-Francisco \& Brangier, 2013; Morschheuser et al., 2017), but most of them operate on a fairly high level, or even on a meta level, and do not describe their operationalization in terms of how learning material would actually be transformed when following these design processes and how it could exactly be enriched with game design elements (Rughinis, 2013). Moreover, these approaches remain theoretical. Furthermore, the number of current design approaches for creating gamified learning arrangements shows that this research field still seeks more standardization (Deterding et al., 2013). An increase in standardization, like IEEE LOM (IEEE, 2009), would contribute to the comparability of gamified learning arrangements and increases the possibility for evaluations that would actually allow new insights on the impact that gamification has on learning.

An initial way to address this problem is to develop design approaches that do not end with applying gamified design processes on learning materials or vice versa, but which aim to integrate further, for example, by supporting the structured and effective implementation in learning management systems. That would bridge the gap between the theory of gamified learning arrangements (including their learning contents) and the implementation in educational software. This software could directly rely on a gamification design framework (e.g. Werbach \& Hunter, 2015) and furthermore would be able to represent common learning concepts like certain types of learning tasks. According to Dicheva \& Dichev (2016) this kind of software is still missing in the prevailing scenery of gamified learning 
platforms. They see a necessity for “... a new type of educational software that can support intelligent mentoring of gamified flipped learning formal classes or informal groups of learners" (Dicheva \& Dichev, 2016, p. 5) and characterize it as being “... easy-to-use tools for automatic generation, checking, and personalized delivery of abundance of practice exercises" (Dicheva \& Dichev, 2016, p. 6).

\subsection{Goals}

In order to tackle this issue, our vision is to overcome this gap between theoretical gamified learning concepts and their practical implementation in the context of a learning management system. To do so, relevant concepts of gamification (e.g. game design elements) for learning purposes had to be identified first. In a second step, their inner structure and interdependencies with other gamification and learning concepts have to be analysed. Third, a technique for systemizing these findings was needed in order to provide a tool which allows the effective implementation of this systemization. Therefore, the following research questions were formulated:

RQ1: Which elements of gamification have recently been applied in higher education learning contexts?

RQ2: How can these gamification elements be described according to their inner structure, interdependencies with further elements and rules they follow?

RQ3: How can the findings of RQ1 and RQ2 be organized and which technique seems suitable for this task?

RQ4: How could a software prototype reflect this organization and allow lecturers to effectively create gamified learning arrangements within a learning management system which can be directly used by learners?

While RQ1-RQ3 have already been discussed and answered in previous work (Bartel \& Hagel, 2016a; Bartel \& Hagel, 2016b; Bartel, Hagel \& Wolff, 2017), this contribution focuses on answering RQ4.

\subsection{Related Work}

Currently, to our best knowledge and after extensively searching the literature there is no comparable approach for the educational field existent.

In the enterprise sector, Herzig, Ameling \& Schill (2012) present an extension for SAP SE which can be used for creating and implementing gamified business-to-consumer (B2C) scenarios with a textual domain-specific language. Hence, the underlying systematization of gamification concepts is expressed using a textual domain-specific language (DSL) that is used for generating code in the SAP SE environment (Herzig et al., 2015). 
Besides this, Yohannis (2016) presents a concept and early results for using a graphical domain-specific language in order to generate a game which supports the learning of software modeling. Since Yohannis' work is still in its early stages, it cannot be determined to what extent software modeling topics are covered through his work and which learning arrangements are supported in particular. Furthermore it is not clear how these modeling games shall be designed by facilitators and how the Lense of Instrinsic Skill Atoms (Deterding, 2015) is actually contributing.

\subsection{Structure}

The rest of the paper is structured into 3 sections. The next section describes the methodological foundations for the research and the results that were gained so far. Section 3 details the implementation and shows off characteristics of the software. The last section summarizes the findings and gives an outlook on future work.

\section{Developing an Executable Domain-Specific Modeling Language}

\subsection{Research Design}

Since the output of this research is at least one artefact, we follow the Design Science Research (DSR) model of research (Hevner et al., 2004). DSR covers two complementary paradigms, behavioral science as well as design science (Hevner et al., 2004, p. 76f.) and relies upon requirements derived from real world problems (human beings, organizations and technology) as well as the proper application of science (foundations and methodologies). Due to the fact that Hevner et al. (2004) do not determine sub-methods for each activity when making use of DSR, we may apply context-specific methods that are adequate for creating and validating a new increment of an artefact. These sub-methods are briefly described in the following.

\subsection{A Brief Summary Regarding the Results of RQ1-RQ3}

For answering RQ1 we have conducted a literature review using a qualitative approach according to Mayring's document analysis method (2015). In total, $n=32$ papers were chosen for evaluation out of a total sample of $N=3656$. We identified a large number of game design elements but reduced them to the following components by abstracting differences and, based on further literature reviews that tried to answer RQ1, and besides focusing on the most widespread: Badges, Points, Levels, Skills and Unlocks. For them, a platform analysis of 11 gamified learning platforms was conducted (RQ2) in order to extract their inner structure (Bartel, Hagel \& Wolff, 2017). The results were documented in domain models. Considering both, the domain models as well as the insights of the literature review, we were able to abstract these concepts and create (RQ3) a graphical domain-specific modeling language (DSML) following the domain-specific modeling 
(DSM) approach by Kelly \& Tolvanen (2008). The parts of the DSML that a facilitator can use for modeling gamified learning arrangements are shown in figure 1. The DSML is based on the Gamified Course Design Process (GCDP) which is a structured method for creating gamifying learning arrangements and uses the same concepts as the DSML (Bartel \& Hagel, 2016b). The GCDP serves as supportive means before the actual gamified learning arrangement is designed with the DSML which allows to systematically create gamified learning arrangements on a theoretical level.

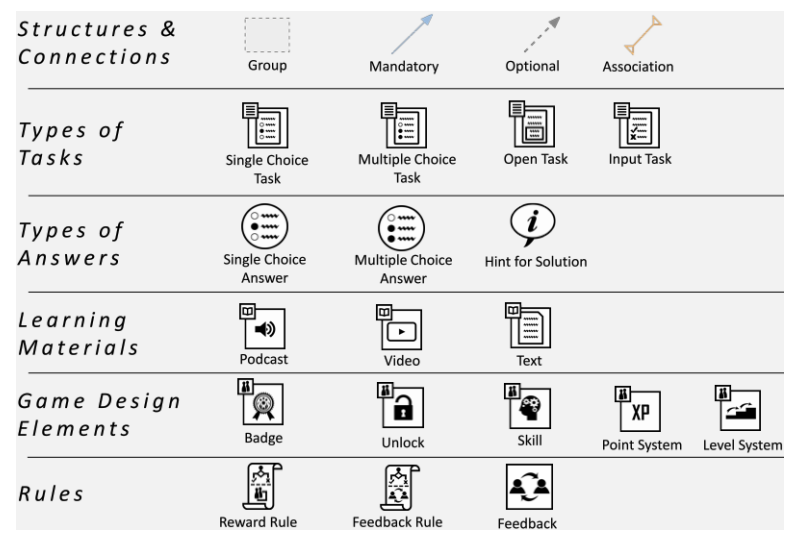

Figure 1. Graphical Notation for the DSML

\section{Implementation}

Figure 2 shows the DSML in action. This modeling example illustrates a single choice task with three answers and one feedback rule. Rules in general consist of a user action that has to be performed to trigger the rule, one or more constraints that have to be fulfilled to be executed further and accordingly one or more consequences that are executed when all constraints are fulfilled. In this case a feedback rule is attached to the single choice task, meaning that the user is notificated with the feedback object of Feedback_13614 when some defined user action is detected on the single choice task. The same procedure can be applied for reward rules, while a reward is considered to be one or more game design elements.

For defining the required information for each graphical element, for example, the question text in a particular task or the user action for triggering a rule, each element provides further input dialogues which can be opened by double-clicking on the element. In this way game mechanics like collecting items or competition (Werbach \& Hunter, 2014) can be implemented just by using rules and structuring the processing procedure of the tasks with the relations. Tasks and learning materials can be connected in two different ways, assuming that A stands for one task or learning material object and B for another: 
1. Mandatory $(\mathrm{A} \rightarrow \mathrm{B})$ : $\mathrm{B}$ is only unlocked for the learner if $\mathrm{A}$ is completed.

2. Optional $(\mathrm{A}-\rightarrow \mathrm{B})$ : The completion of $\mathrm{A}$ is not necessary for working on $\mathrm{B}$, but recommended.

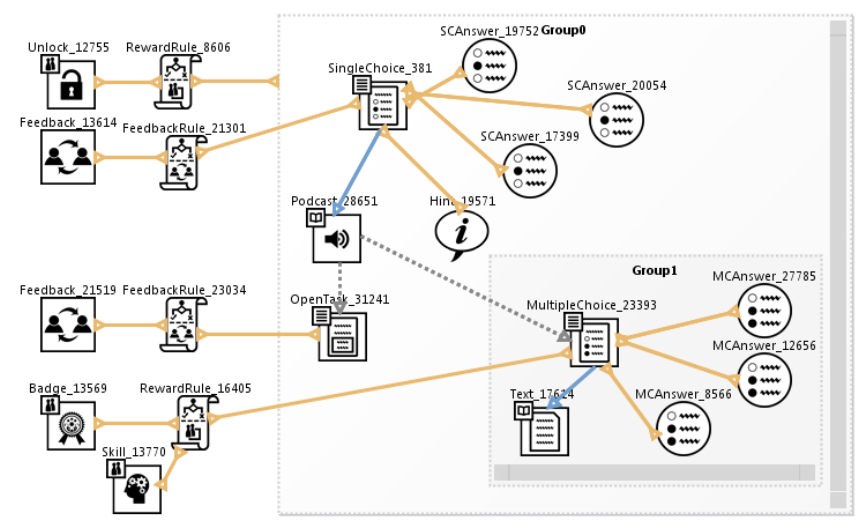

Figure 2. The DSML in Action

Hence, in our example, only when the single choice task is completed, the learner can listen to a podcast. After that, a learner can decide whether to continue with the open task or the multiple choice task. Thus, the concept of learning paths is also provided. For creating more complex reward or feedback rules, tasks or learning materials can be clustered in groups. Groups also allow the attachment of rules. Therefore it is for example possible to define a rule for a group, which is triggered on every completion of task within that group that checks if all single choice tasks are answered correctly and in time and if so rewards learners with a badge and a certain amount of a user-defined point currency.

Once a facilitator has completed the model of the gamified learning arrangement, one is able to automatically generate a learning management system out of the model, which learners can use for actually working on materials or tasks, meaning that a facilitator does not need to write any source code. Due to the way the DSML is constructed, one is easily able to extend the DSML, for example, by adding a new type of rule or game design element. That allows a high degree of flexibility for lecturers and does not force existing gamified learning arrangements to be implemented in learning management systems, where they do not fit and therefore require a lot of compromises which can reduce their quality. Furthermore the models of the designed gamified learning arrangements are exchangeable between system environments, since the underlying technology is decoupled from the contents of the model. 


\section{Conclusion and Future Work}

The implementation of the learning management system is still ongoing and not finished yet. It is also planned to integrate an analytics component for lecturers, which tracks the individual learning progress of learners and provides extension possibilities for approaches out of the adaptive learning theory.

As soon as the prototype is completed, it is planned to evaluate it from both sides, the design view focusing on lecturers, and the learning view while learners are focused. It is assumed that the results of the evaluation will confirm our point of view on the entire concept and thus adds value to the research community in this research field.

\section{References}

Bartel, A., \& Hagel, G. (2016a). Gamified Just-in-Time Teaching - A Conceptual Approach Based on Best Practices. In G. Hagel \& J. Mottok (Eds.), Proceedings of ECSEE 2016 European Conference Software Engineering Education (pp. 1-17). Aachen: Shaker.

Bartel, A., \& Hagel, G. (2016b). Gamifying the Learning of Design Patterns in Software Engineering Education. In Global Engineering Education Conference (EDUCON), 2016 IEEE (pp. 74-79). Los Alamitos: IEEE.

Bartel, A., Hagel, G., \& Wolff, C. (2017). Towards a Generic Platform for Implementing Gamified Learning Arrangements in Engineering Education. In Global Engineering Education Conference (EDUCON), 2017 IEEE. Los Alamitos: IEEE.

Danelli, F. (2015). Implementing Game Design in Gamification. In T. Reiners \& L. C. Wood (Eds.), Gamification in Education and Business (pp. 67-79). Cham: Springer.

Dicheva, D., \& Dichev, C. (2016). An Active Learning Model Employing Flipped Learning and Gamification Strategies. In Proceedings of first Int. Workshop on Intelligent Mentoring Systems (IMS 2016), Zagreb, Croatia (pp. 1-6).

Deterding, S. (2015). The Lens of Intrinsic Skill Atoms: A Method for Gameful Design. Human-Computer Interaction, 30(3-4), 294-335.

Deterding, S., Björk, S. L., Nacke, L. E., Dixon, D., \& Lawley, E. (2013). Designing gamification: creating gameful and playful experiences. In W. Mackay, S. Brewster, \& S. Bødker (Eds.), Extended Abstracts on Human Factors in Computing Systems (pp. 3263-3266). New York: ACM.

Deterding, S., Dixon, D., Khaled, R., \& Nacke, L. (2011). From game design elements to gamefulness: defining gamification. In A. Lugmayr, H. Franssila, C. Safran, \& I. Hammouda (Eds.), Proceedings of the 15th International Academic MindTrek Conference: Envisioning Future Media Environments (pp. 9-15).

Herzig, P., Ameling, M., \& Schill, A. (2012). A Generic Platform for Enterprise Gamification. In Joint Working IEEE/IFIP Conference on Software Architecture (WICSA) and European Conference on Software Architecture (ECSA) (pp. 219-223).

Herzig, P., Ameling, M., Wolf, B., \& Schill, A. (2015). Implementing Gamification: Requirements and Gamification Platforms. In T. Reiners \& L. C. Wood (Eds.), Gamification in Education and Business (pp. 431-450). Cham: Springer Publishing. 
Hevner, A. R., March, S. T., Park, J., \& Ram, S. (2004). Design Science in Information Systems Research. MIS Q, 28(1), 75-105.

IEEE (2009). IEEE Standard for Learning Object Metadata. (1484.12.1-2002).

Kapp, K. M., Blair, L., \& Mesch, R. (2014). The Gamification of Learning and Instruction Fieldbook: Ideas into Practice. San Francisco: John Wiley \& Sons.

Kelly, S., \& Tolvanen, J.-P. (2008). Domain-specific modeling: Enabling full code generation. Hoboken, N.J.: John Wiley \& Sons.

Marache-Francisco, C., \& Brangier, E. (2013). Process of Gamification. From the Consideration of Gamification to its Practical Implementation. In CENTRIC 2013, The Sixth International Conference on Advances in Human oriented and Personalized Mechanisms, Technologies, and Services (pp. 126-131).

Mayring, P. (2015). Qualitative Inhaltsanalyse: Grundlagen und Techniken (12th ed.). Weinheim: Beltz.

Morschheuser, B., Werder, K., Hamari, J., \& Abe, J. (2017). How to gamify? A method for designing gamification. In Proceedings of the 50th Hawaii International Conference on System Sciences (HICSS) (1298-1307). Los Alamitos: IEEE.

Nah, F. F.-H., Zeng, Q., Telaprolu, V. R., Ayyappa, A. P., \& Eschenbrenner, B. (2014). Gamification of Education: A Review of Literature. In F. F.-H. Nah (Ed.), HCI in Business: First International Conference, HCIB 2014, Heraklion, Crete, Greece, June 22-27, 2014. Proceedings (pp. 401-409). Cham: Springer.

Rughinis, R. (2013). Flexible Gamification in a Social Learning Situation: Insights from a collaborative review exercise. In N. Rummel, M. Kapur, M. Nathan, \& S. Puntambekar (Eds.), 10th International Conference on Computer Supported Collaborative Learning CSCL 2013. Conference Proceedings Volume II (pp. 137-140). International Society of the Learning Sciences (ISLS).

Werbach, K., \& Hunter, D. (2015). The Gamification Toolkit: Dynamics, Mechanics, and Components for the Win. New York: Wharton Digital Press.

Yohannis, A. (2016). Gamification of Software Modelling Learning. In S. Nejati \& R. Salay (Eds.), Proceedings of the Doctoral Symposium at the 19th ACM/IEEE International Conference of Model-Driven Engineering Languages and Systems 2016 (pp. 1-7). 